\title{
The Combination of Electron Capture Dissociation and Fixed Charge Derivatization Increases Sequence Coverage for $O$-Glycosylated and $O$-Phosphorylated Peptides
}

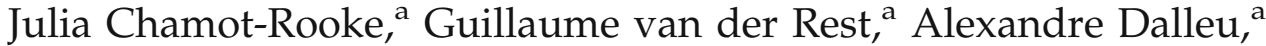 \\ Sylvie Bay ${ }^{c}$ and Jérôme Lemoine ${ }^{b}$ \\ ${ }^{a}$ Laboratoire des Mécanismes Réactionnels, Ecole Polytechnique, CNRS, Palaiseau, France \\ b UMR 5180 Sciences Analytiques (Université Lyon 1 et CNRS), Villeurbanne, France \\ c Unité de Chimie Organique, Institut Pasteur CNRS, Paris, France
}

Electron capture dissociation (ECD) has become an alternative method to collision-activated dissociation (CAD) to avoid gas-phase cleavage of post-translational modifications carried by side chains from the peptide backbone. Nonetheless, as illustrated herein by the study of $O$-glycosylated and $O$-phosphorylated peptides, the extent of ECD fragmentations may be insufficient to cover the entire peptide sequence and to localize accurately these modifications. The present work demonstrates that the derivatization of peptides at their $\mathrm{N}$-terminus by a phosphonium group improves dramatically and systematically the sequence coverage deduced from the ECD spectrum for both O-glycosylated and O-phosphorylated peptides compared with their native counterparts. The exclusive presence of N-terminal fragments (c-type ions) in the ECD spectra of doubly charged molecular cations simplifies peptide sequence interpretation. Thus, the combination of ECD and fixed charge derivatization appears as an efficient analytical tool for the extensive sequencing of peptides bearing labile groups. (J Am Soc Mass Spectrom 2007, 18, 1405-1413) (c) 2007 American Society for Mass Spectrometry

$\mathrm{T}$ The field of proteomics usually aims at differential characterization of the complete pattern of protein expression, for instance between cells in a normal state and those in a pathological state. Protein identification is now routinely achieved on a large scale (tens or hundreds of proteins per day) by combining enzymatic digestion, mass spectrometric analysis of the resulting peptides, and subsequent database search. If few peptides or short peptide sequences are sufficient for protein identifications into databases, a complete determination of the primary structure is nevertheless useful to detect modifications that can be involved in the regulation of protein function. Indeed, the catalytic property, the conformation, the turnover, or the subcellular localization of proteins are largely under the control of various peptide sequence processings named posttranslational modifications. These modifications (PTM) involve the proteolytic maturation of the proteins and/or the transfer of chemical groups (phosphorylation, glycosylation, acetylation, sumoylation) on specific amino-acids (serine, threonine, or tyrosine

Address reprint requests to Dr. Julia Chamot-Rooke, Laboratoire des Mécanismes Réactionnels, Ecole Polytechnique, CNRS, 91 128, Palaiseau, France;. E-mail: julia.chamot-rooke@polytechnique.edu for O-phosphorylations, serine or threonine for $\mathrm{O}$ glycosylations, asparagine for $\mathrm{N}$-glycosylations). The most obvious strategy for mapping the modifications along the peptide chain involves a comparison between the sequence obtained by mass spectrometric data and the one deduced from the open reading frame coded by the gene sequence. However, a drawback arises from the propensity of groups conjugated to the amino acid side chains to dissociate readily upon collisional activation. For instance, collisionally-activated dissociation (CAD) MS/MS spectra of protonated precursor ions of phosphorylated peptides exhibit intense ions originating from the elimination of metaphosphoric $\left(\mathrm{HPO}_{3}\right.$, loss of $\left.80 \mathrm{Da}\right)$ or orthophosphoric acid $\left(\mathrm{H}_{3} \mathrm{PO}_{4}\right.$, loss of $\left.98 \mathrm{Da}\right)$ [1]. In the case of $\mathrm{O}-\mathrm{N}$-acetylgalactosamine and $\mathrm{O}-\mathrm{N}$-acetylglucosamine substituted peptides, singly protonated molecular ions undergo a predominant neutral loss of $203 \mathrm{Da}$, and doubly charged peptides [2,3] form an intense fragment ion at $\mathrm{m} / \mathrm{z} 204$. These favored gasphase eliminations can help the specific detection of modified peptides by reconstructing ion chromatograms either on specific neutral losses or diagnostic ions. For peptides containing several amino acids potentially modified (serine, threonine), the precise localization of the modification remains a difficult 
task since the fragment ions bearing the modification are often lacking or are of low abundance in the MS/MS spectra.

To overcome the limitations of CAD-MS/MS, numerous strategies have been proposed to localize phosphate, $O$-GalNAc, and O-GlcNAc moieties $[4,5]$ that combine $\beta$-elimination and mass spectrometric characterization of end products. However, these protocols still suffer from some shortcomings. In particular, both the temperature and duration of the $\beta$-elimination reaction have to be carefully adjusted to each sample to avoid nonspecific Ser or Thr dehydration.

Electron capture dissociation (ECD) [6, 7] and, more recently, electron-transfer dissociation (ETD) [8] have emerged as powerful novel dissociation techniques that do not induce fragmentation of labile bonds between conjugated groups and peptides. Basically, the dissociation of the reduced cation species $[\mathrm{M}+n \mathrm{H}]^{(n-1)+}$. leads to a series of fragment ions of the $c$ and $z$ type. The interest of ECD has now been widely illustrated for the unambiguous assignment of phosphorylation [9], O-glycosylation [6], or $\gamma$-carboxylation sites [10], as well as for intact protein sequencing in the socalled top-down approach [11, 12]. It is now established that the electron capture is followed by the formation of long-lived intermediate complexes in which hydrogen rearrangements can take place [13]. These $c / z$ hydrogen-bonded complexes can further dissociate to give "classical" $c / z$ ions or related species such as $\mathrm{z}^{\prime}$ or $\mathrm{z}^{\prime \prime}$ ions if hydrogen atom transfers have taken place before the dissociation [14]. For large proteins, a disruption of the weak intramolecular noncovalent bonds that may prevent the separation of the ECD products is often necessary $[15,16]$. This can be done by "activated ion ECD (AI-ECD)" in which the parent ions are collisionally- or IR-heated during electron irradiation. AI-ECD employing laser irradiation following electron capture has also proved to be an efficient way to obtain a substantially higher number of fragments than regular ECD for phosphopeptides [17] or $\mathrm{N}$-glycopeptides [18].

In an alternative approach, phosphate [19] or glycan moieties [20] have been localized by CADMS/MS or postsource decay analysis of peptides derivatized at their N-termini with a phosphonium group. It has been shown that when a singly charged cation was selected as an ion precursor, loss of the conjugated moiety could be almost completely suppressed. This feature is likely due to the lack of a mobile proton able to drive a charge-induced fragmentation mechanism.

The present work deals with an evaluation of ECD combined to fixed-charge derivatization in the perspective of a general strategy that would allow concomitantly the localization of modification sites and extensive sequence coverage, ideally from a unique series of fragment ions.

\section{Experimental}

\section{Nano-Electrospray Mass Spectrometry}

All experiments were carried out with a 7-T APEX III FT-ICR mass spectrometer (Bruker Daltonik GmbH, Bremen, Germany) equipped with a 7 tesla actively shielded superconducting magnet, an infinity cell [21], ${ }^{\circ}$ and $^{\circ} a^{\circ}$ nano-electrospray ${ }^{\circ}{ }^{\circ}{ }^{\circ}{ }^{\circ}$ source. ${ }^{\circ}$ Nano-electrospray needles were purchased from Proxeon (Odense, Denmark) and filled with $2 \mu \mathrm{L}$ of the peptide solution $(2 \mathrm{pmol} / \mu \mathrm{L}$ in $50 \% \mathrm{MeOH} /$ water/ $0.2 \%$ formic acid). The needles were subsequently opened by breaking the tapered end of the tip under a microscope. A stable spray was observed by applying a voltage of about $-700 \mathrm{~V}$ between the needle (grounded) and the entrance of the glass capillary used for ion transfer. A source temperature of $50^{\circ} \mathrm{C}$ was used for all nano-electrospray experiments. The estimated flow rate was $20-50 \mathrm{~nL} / \mathrm{min}$. Ions were stored in the source region in a hexapole guide for $1 \mathrm{~s}$ and pulsed into the detection cell through a series of electrostatic lenses. Ions were finally trapped in the cell using either gas-assisted dynamic trapping ( $\mathrm{Xe}$ pulses, upper pressure around $10^{-7} \mathrm{mbar}$ ) with front and back trapping voltages of 3.0 and $3.5 \mathrm{~V}$ for trapping, reduced to 0.9 and $0.95 \mathrm{~V}$ for detection or static trapping. Mass spectra were acquired from $\mathrm{m} / \mathrm{z}$ 500 to 3000 with $512 \mathrm{k}$ data points and monoisotopic peaks were automatically labeled using the XMASS 6.1.4 (Bruker Daltonik GmbH, Bremen, Germany) software. For Sustained Off Resonance Irradiation Collision ${ }^{\circ}$ Activated ${ }^{\circ}$ Dissociation ${ }^{\circ}(S O R I-C A D)^{\circ}[22]^{\circ} \mathrm{ex}-$ periments, the ion to fragment was isolated by radio frequency (rf) ejection of all unwanted ions using both low-voltage single rf pulses (soft shots) at their resonance frequencies and a chirp excitation covering the region of interest. The fragmentation was performed using an excitation duration of $400 \mu \mathrm{s}\left(\mathrm{V}_{p-p}=\right.$ $37 \mathrm{~dB}$ ) at a frequency $500 \mathrm{~Hz}$ higher than the cyclotron frequency of the ion of interest. Xenon was used as the collision gas and introduced several times through a pulsed valve to get a pressure in the cell up to $10^{-7} \mathrm{mbar}$. The excitation/detection of all fragment ions was performed after a $4 \mathrm{~s}$ pumping delay. For ECD experiments, a hollow electron emitter was used $^{\circ}[23]^{\circ}{ }^{\circ}$ The $^{\circ}$ emitter $^{\circ}$ generates ${ }^{\circ} a^{\circ}$ hollow, ${ }^{\circ}$ tubular electron beam and was heated with a 1.9 A current. Isolated multiply charged ions were irradiated during $150 \mathrm{~ms}$ with electrons having $<1 \mathrm{eV}$ kinetic energy. An external calibration using $\mathrm{NaI}(2 \mathrm{mg} / \mathrm{mL}$ in isopropanol/water) was done daily.

\section{Synthetic Glycopeptides and Phosphopeptides}

Glycopeptide syntheses were performed manually using standard Fmoc chemistry protocols on a polystyrene resin functionalized with $p$-benzyloxybenzyl alcohol and esterified with the C-terminal residue (alanine 
Table 1. Primary structure and molecular masses (in Da) before and after TMPP derivatization (+ 573.18841 Da) of all glycopeptides and phosphopeptides studied

\begin{tabular}{lclcc}
\hline \multicolumn{1}{c}{ Sequence } & Peptide designation & Modification & Mr before derivatization & Mr after derivatization \\
\hline \hline ALGST*T*PPA & G1 & $N$-acetylgalactosamine & 1219.5820 & 1792.77041 \\
ALGS*T*TPPA & G2 & $N$-acetylgalactosamine & 1219.5820 & 1792.77041 \\
ALGS*TT*PPA & G3 & $N$-acetylgalactosamine & 1219.5820 & 1792.77041 \\
ALGS*T*T*PPA & G4 & $N$-acetylgalactosamine & 1422.6614 & 1995.84981 \\
GTTPSPVPT*TSTTSAPG & G5 & $N$-acetylgalactosamine & 1760.8316 & 2334.02001 \\
KGGGTTSTT*SAPG & G6 & $N$-acetylgalactosamine & 1323.6154 & 1896.80381 \\
YLEKRAS*GQAFE & P1 & Phosphate & 1477.6603 & 2050.84871 \\
YFELILS*PRSKE & P2 & Phosphate & 1560.7589 & 2133.94731 \\
YPEFPLS*KKK & P3 & Phosphate & 1315.6577 & 1888.84615 \\
YSDSPS*TST & P4 & Phosphate & 1023.3434 & 1596.5324
\end{tabular}

The asterisk $\left({ }^{*}\right)$ indicates the localization of the post-translational modifications.

or glycine). Briefly, the protected amino-acids and the glycosylated building blocks [Fmoc-Ser $(\alpha-$ GalNAc $\left.\{\mathrm{OAc}\}_{3}\right)-\mathrm{OH}$ or Fmoc-Thr $\left(\alpha-\mathrm{GalNAc}\{\mathrm{OAc}\}_{3}\right)-$ $\mathrm{OH}$ ] (3 equivalents) were incorporated in the peptide chain using 2-(1H-benzotriazole-1-yl)-1,1,3,3-tetramethyluronium tetrafluoroborate (TBTU)/1-hydroxybenzotriazole (HOBT)/di-isopropylethylamine (DIEA) as the coupling reagent. Fmoc protection was removed with $20 \%$ piperidine in DMF. The products were cleaved from the resin with aqueous trifluoroacetic acid $(95 \%)$. Deacetylation of the sugar residue was achieved with a catalytic amount of sodium methoxide in methanol at $\mathrm{pH}$ 11. The glycopeptides were purified by reverse phase high-performance liquid chromatography using a Perkin-Elmer pump system with a UV detector at $230 \mathrm{~nm}$. The column was a Waters Delta Pak C18 $(15 \mu, 300 \AA, 7.8 \times 300 \mathrm{~mm})$ and the gradient was performed with water $(0.1 \%$ TFA)/acetonitrile (from $0 \%$ to $30 \%$ ) over $20 \mathrm{~min}$. The compounds were charac- terized by amino acid analysis (AAA) and electrospray mass spectrometry (ESMS).

\section{N-Terminus Modified Acetylphosphonium Derivatives}

To a solution of a peptide (20-100 pmol) in $2 \mu \mathrm{L}$ acetonitrile/water (2/8) (vol/vol) was added $2 \mu \mathrm{L}$ of $50 \mathrm{mM}$ Tris- $\mathrm{HCl}$ buffer ( $\mathrm{pH}$ 8.2) followed by $1 \mu \mathrm{L}$ of $1 \mathrm{mM}$ (N-succinimidyloxycarbonylmethyl)tris(2,4,6-trimethoxyphenyl)phosphonium bromide (Sigma-Aldrich, SaintQuentin Fallavier, France). The mixture was vortexed for $30 \mathrm{~s}$ and then sonicated in a water bath for $30 \mathrm{~min}$ (final bath temperature $<30^{\circ} \mathrm{C}$ ). The resulting mixture was evaporated (centrifugal evaporator) to dryness and the residue redissolved in $50 \% \mathrm{MeOH} /$ water $/ 0.2 \%$ formic acid. The derivatives were stored at $-20^{\circ} \mathrm{C}$ and further analyzed directly by nano-electrospray.

Table 2. $\mathrm{c}$ and $\mathrm{z}$ ions cleavages observed for ECD experiments performed on doubly charged native and phosphonium derivatized peptides (except $\mathrm{P} 3$ for which triply charged ions were selected)

\begin{tabular}{|c|c|c|}
\hline Peptide & ECD fragment ions on the native peptide & ECD fragment ions on the phosphonium derivatized peptides \\
\hline G1 & A L G S T T T* P PlA & 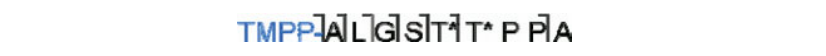 \\
\hline G2 & AL G SATTTT P PlA & TMPPIA]LIG]S $S^{*} T^{*} \mid T$ P PlA \\
\hline G3 & A L G S SiThT* P PlA & 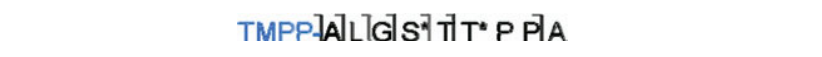 \\
\hline G4 & $A L G S^{A} T^{*} T^{*} P P \mid A$ & 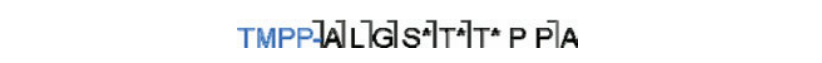 \\
\hline G5 & G TLT P S P V P T'TTSTITINSIA PIG & TMPPIGlTit Pls Plv PlT^ITlsititisla Plg \\
\hline G6 & 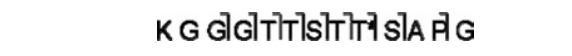 & 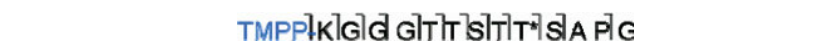 \\
\hline P1 & 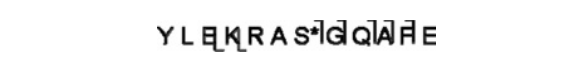 & 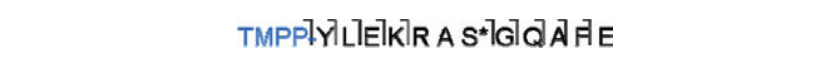 \\
\hline $\mathbf{P 2}$ & 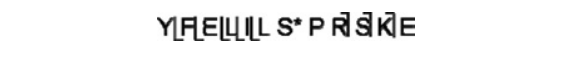 & 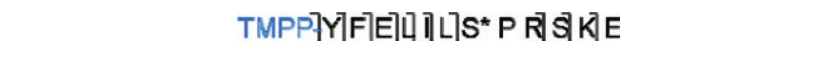 \\
\hline P3 & 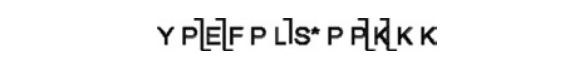 & 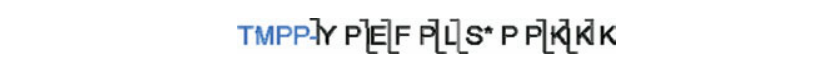 \\
\hline $\mathbf{P 4}$ & YSDSPIS耳TIST & 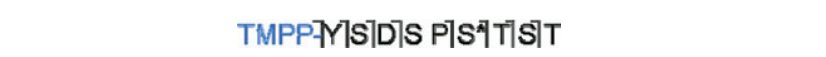 \\
\hline
\end{tabular}

The asterisk $\left({ }^{*}\right)$ indicates the localization of the post-translational modifications in the peptide sequence. 


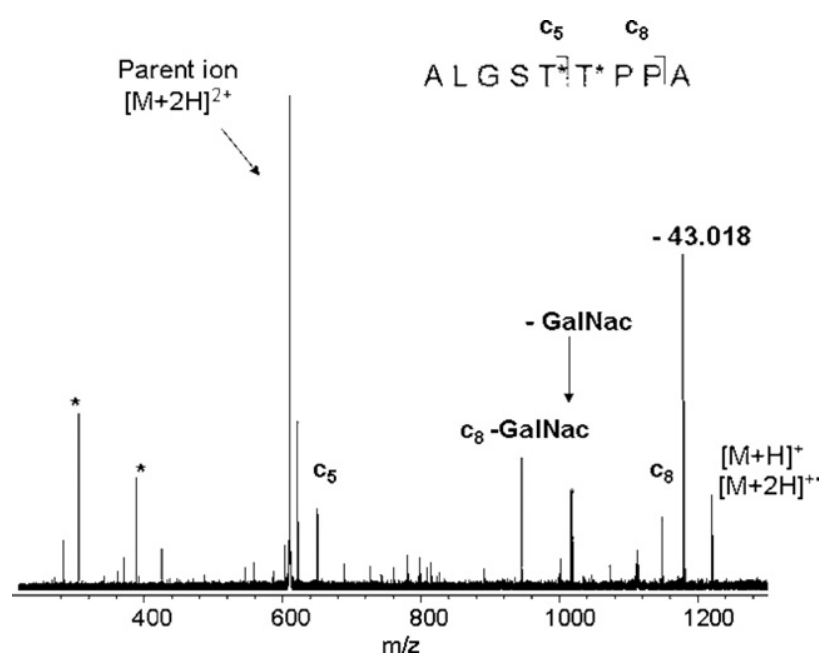

Figure 1. ECD of $2^{+}(m / z$ 610.7983) of the glycosylated peptide G1 (two GalNAc groups).

\section{Results and Discussion}

As expected, all protonated glycopeptides and phosphopeptides ${ }^{\circ}$ under $^{\circ}$ study $^{\circ}\left(\text { Table }^{\circ} 1\right)^{\circ}$ undergo ${ }^{\circ}$ an intense loss of the PTM (data not shown) when subjected to SORI-CAD. The lability of these PTM groups is well known and is induced by a preferential transfer of a mobile proton to the sugar-peptide glycosidic bond or to the phosphoester bond.

\section{ECD Analysis of Native Peptides}

ECD results for all peptides under study are gathered in the ${ }^{\circ}$ Table $2 .{ }^{\circ}$ For ${ }^{\circ}$ all ${ }^{\circ}$ glycopeptides ${ }^{\circ}$ and ${ }^{\circ}$ for ${ }^{\circ}$ phosphopeptides P1, P2, and P4, the doubly charged ions were isolated and irradiated with electrons. For the phosphopeptide $\mathbf{P 3}$, the triply charged species was much more intense and was chosen for ECD experiments.

\section{Native Glycopeptides}

Although ECD is often very useful for glycosylation site localization, for all glycopeptides under consideration, ECD experiments on the most intense doubly charged ions yielded an incomplete $c$ ions series and nearly no $z$ ion. The ECD spectrum of the $\left(2^{+}\right)$ion of diglycosylated peptide ${ }^{\circ} \mathbf{G 1}$ is $^{\circ}$ Shown $^{\circ}$ in ${ }^{\circ}$ Figure $^{\circ} 1 .^{\circ}{ }^{\circ}$ Clearly ${ }^{\circ}$ the ${ }^{\circ}$ glycosylation site cannot be assigned on the basis of this spectrum since one GalNAc could be either on Ser3 or on Thr4.

A similar fragmentation pattern was obtained for all glycopeptides lacking a basic amino acid (G1-G5). For peptides lacking amino acids of high gas-phase basicity (arginine, lysine, histidine), such as peptides G1-G5, the preferential formation of $c$ ions (versus $z$ ions) upon

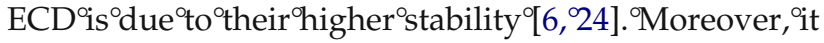
has been shown that this kind of peptide has often a nonclassical behavior upon ECD, a common feature being the formation of $b$ ions. It was demonstrated by
Cooper $[25]^{\circ}{ }^{\circ} d^{\circ}$ confirmed ${ }^{\circ}$ very ${ }^{\circ}$ recently ${ }^{\text {by }}{ }^{\circ}$ Haselmann

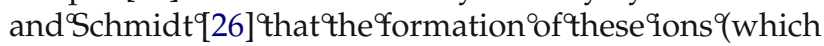
are true $\mathrm{N}$-terminal $b$-type ions) can be explained by typical MS/MS pathways (low-energy threshold backbone dissociation) from the even-electron chargedreduced cation. These $b$ ions were not detected in the ECD spectra of all glycopeptides under study. Conversely, an important fragmentation observed for peptides G1-G4 (and to a lesser extent G5 and G6) is a loss of GalNAc (loss of 203.079 Da from [MH] ${ }^{+}$ions). This fragmentation (which is the major one in SORI-CAD experiments) can be explained like the formation of $b$ ions, i.e., via the mobile proton pathway from the $[\mathrm{MH}]^{+}$ions formed by hydrogen loss. Another explanation could be a vibrational excitation of the precursor ions due to collisions with the trapping gas still residing in the ICR cell. This is unlikely since experiments realized with static trapping gave the same results. Moreover, a vibrational excitation of selected ions followed by ECD would have led to AI-ECD experiments and numerous backbone cleavages. As mentioned above, ECD spectra obtained for all glycopeptides under study exhibited only a few fragmentations, which excludes the possibility of important vibrational excitation.

Ions corresponding to either the loss of $43.018 \mathrm{Da}$ $\left(\mathrm{C}_{2} \mathrm{H}_{3} \mathrm{O}\right)$ from the radical cationic species or $42.016 \mathrm{Da}$ $\left(\mathrm{C}_{2} \mathrm{H}_{2} \mathrm{O}\right)$ from the even electron species $[\mathrm{M}+\mathrm{H}]^{+}$are observed in all spectra. This fragmentation has been discussed ${ }^{\circ}$ in $^{\circ}$ details $^{\circ}$ by $^{\circ}$ Mormann $^{\circ}$ et $^{\circ}$ al..$^{\circ}[27]^{\circ}$ who ${ }^{\circ}$ proposed a mechanism based on the attack of a hot hydrogen atom at the nitrogen of the $\mathrm{N}$-acetyl group of one glycan leading to an N-centered hypervalent radical, which could subsequently fragment by loss of an acetyl radical (43.0178 Da). Nevertheless, it should be noted that the attack of the hydrogen atom should be more likely at the carbonyl oxygen which has a higher hydrogen affinity than the amide nitrogen and therefore this mechanism seems unlikely. Other mechanisms involving, as the first step, either a direct electron capture by one amide OCN $\pi^{*}$ orbital from the peptide backbone followed by a facile cleavage of the $\mathrm{N}-\mathrm{C}_{\alpha}$ bond'(superbase mechanism) $\left[28^{\circ}-30\right]^{\circ}$ or through a bond electron-transfer from one of the Rydberg orbital of the hypervalent species formed by the attachment of the electron ${ }^{\circ} t^{\circ} a^{\circ}$ positively ${ }^{\circ}$ charged $^{\circ}$ site $[31,32]^{\circ}$ may $^{\circ}$ play $^{\circ}$ a role here. The elimination of neutral ketene as a secondary fragmentation of the reduced species can be excluded since SORI-CAD of the $[\mathrm{M}+\mathrm{H}]^{+}$species (selected before the electron capture) does not give any loss of $42 \mathrm{Da}$. Careful analysis of the experimental data on this set of glycopeptides does not lead to any indication on which mechanism is involved in this process.

Another fragmentation observed for glycopeptides G1-G4 is a loss of the GalNAc moiety from $c_{8}$. This fragmentation was first thought to be a water loss from a $z$ ion, but an internal calibration of all ECD spectra definitely rejected this hypothesis. Such glycan loss has been observed mainly from $(z+1 \mathrm{H})$ type fragment ions 


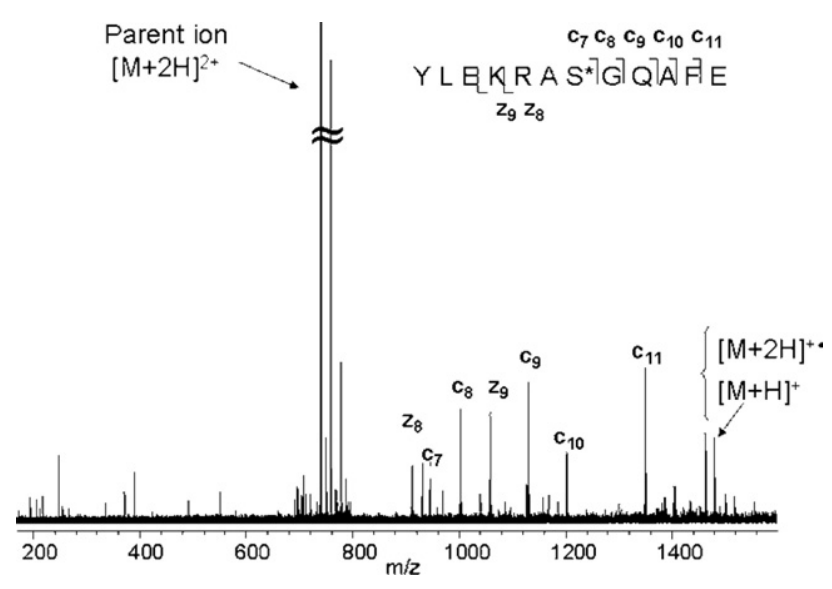

Figure 2. ECD of $2^{+}(\mathrm{m} / \mathrm{z}$ 739.8374) of the phosphorylated peptide $\mathbf{P 1}$.

but $^{\circ}$ also $^{\circ}$ from $^{\circ} \mathrm{C}$ ions $^{\circ}\left[6,{ }^{\circ} 28\right] .^{\circ}$ The $^{\circ}$ formation ${ }^{\circ}$ of ${ }^{\circ}$ this $\left(c_{8}\right.$-GalNAc) intense ion can be explained by two different mechanisms. The first one is a direct loss of GalNac (low-energy pathway) from energetic $c_{8}$ ions or the formation of $c_{8}$-GalNAc is consecutive to the direct loss of GalNac from $[\mathrm{M}+2 \mathrm{H}]^{+}$.

For the glycopeptide G5, which contains one GalNac and no basic amino acid, half of the sequence (Cterminal moiety) can be retrieved in ECD experiments based on the presence of $c$ ions.

Finally, the glycopeptide G6, which contains one GalNAc and one lysine as the N-terminal amino acid exhibits a rather common behavior upon ECD with an acceptable sequence coverage due to the exclusive presence of $c$ ions. Note that among these $c$ ion series, $c_{3}-c_{7}$ isotopic patterns indicate a mixture of $c$ and $c-\mathrm{H}$ ions.

Taken together, these results show that two major factors influence the quality of the ECD spectra in term of sequence coverage. First, the peptide sequence and more particularly the presence of a basic amino acid such as arginine, lysine, or histidine influences the fragmentation pattern observed upon ECD. Indeed, we confirm in this study that peptides that do not contain any basic amino acids yield fragmentations due mainly to the mobile proton pathway (loss of the PTM in our case), ${ }^{\circ}$ as $^{\circ}$ already ${ }^{\circ}$ proposed ${ }^{\circ}$ by $^{\circ}$ Cooper $^{\circ}[25]^{\circ}$ and $^{\circ}$ Haselmann $^{\circ}$ and ${ }^{\circ} S c h m i d t^{\circ}[26] .{ }^{\circ}$ Second, ${ }^{\circ}$ the ${ }^{\circ}$ proximity ${ }^{\circ}$ of ${ }^{\circ}$ the glycans probably induces a steric hindrance which can preclude a hydrogen migration to the peptidic bond (necessary for the "Cornell" mechanism but not for the "superbase" one).

\section{Native Phosphopeptides}

The results are far better than for the glycopeptides since for all phosphopeptides studied the sequence coverage is sufficient to localize the phosphorylation site. Note that the phosphopeptides P1-P3, which give the highest sequence coverages, contain at least two basic amino acids. Nevertheless, all fragment ions are of low abundance and no complete $c$ or $z$ ion series can be obtained. The ECD spectrum of phosphopeptide P1, which does not contain any proline residue and should thus give a whole sequence coverage, is depicted in Figure $^{\circ} 2$.

For this sample, the analysis of $c$ and $z$ ions clearly indicates that the remaining proton remains on the most basic residue (arginine). The same conclusion can be drawn for P2. Neither loss of phosphate nor $b$ ions is observed. For P4, which does not contain any basic amino acids, an abundant loss of one and two molecules of water is observed from $[\mathrm{M}+\mathrm{H}]^{+}$ions and also from $c_{7}$ and $c_{8}$ ions. The formation of these dehydrated $c$ ions can be rationalized by the formation of intact $c$ ions that lose consecutively one or two molecules of water. An alternative mechanism would be the formation of $\left[\mathrm{MH}_{2}^{+\cdot}-\mathrm{H}_{2} \mathrm{O}\right]$ ions, which could yield dehydrated $c$ ions. The absence of $\left[\mathrm{MH}_{2}{ }^{+}\right]$and $\left[\mathrm{MH}_{2}{ }^{+}{ }^{-}\right.$ $\mathrm{H}_{2} \mathrm{O}$ ] ions in the ECD spectrum does not favor this latter mechanism. Note that the sequence of $\mathbf{P 4}$ is rich in hydroxyl functions, which explain the facile loss of water molecules.

These results on both glycopeptides and phosphopeptides show that the localization of labile PTM by ECD can sometimes be difficult. We show in this study that this is especially the case for peptides lacking basic amino acids and it is highlighted when several modifications are close to each other in the sequence. For that reason, the peptides displaying these features were chosen as good candidates to develop ECD after fixedcharge derivatization.

\section{ECD Analysis of Charge Derivatized (TMPP) Peptides}

It has been shown, some years ago, that the addition of a fixed charge group to the $\mathrm{N}$-terminus of a peptide can create important changes in its fragmentation patterns [33]. ${ }^{\circ}$ For $^{\circ}$ example, ${ }^{\circ}$ the ${ }^{\circ}$ use $^{\circ}$ of $^{\circ} \mathrm{TMPP}^{-a_{\text {acetic }}}{ }^{\circ}$ acid $^{\circ} \mathrm{N}$ hydroxysuccinimide ester (TMPP-AcOSu) allows the formation of peptides containing a highly stabilized phosphonium cation on their N-terminus. This charge derivatization produces better fragmentation and simpler spectra during collision activated dissociation experiments since an exclusive and often complete series of $^{\circ} \mathrm{N}$-terminal ${ }^{\circ}$ fragment ${ }^{\circ}$ ions $^{\circ}$ is ${ }^{\circ}$ obtained $^{\circ}[34] .^{\circ}$ This derivatization procedure has also been applied successfully ${ }^{\circ}$ to $^{\circ}$ tryptic $^{\circ}$ digests $^{\circ}[35] .{ }^{\circ}$ In $^{\circ}$ the $^{\circ}$ case $^{\circ}$ of ${ }^{\circ}$ MALDI experiments, where no additional proton is available that precludes the "mobile proton" model, this can be explained ${ }^{\circ}$ by $^{\circ}$ charge-remote $^{\circ}$ fragmentation ${ }^{\circ}\left[36,{ }^{\circ}\right.$ 37]. Very recently, coupling of TMPP derivatization with C-terminal digestion was shown to improve peptide de novo ${ }^{\circ}$ sequencing ${ }^{\circ}$ by $^{\circ} \mathrm{MALDI}^{\circ} \mathrm{MS}^{\circ}[38]$.

The same derivatization procedure has been applied to phosphorylated peptides further analyzed in an ion trap using ${ }^{\circ}$ electrospray ${ }^{\circ}$ as the 9 ionization ${ }^{\circ}$ source [14]. The authors showed that some $a$ and $b$ ions obtained in the MS/MS experiments still contain the phosphate 


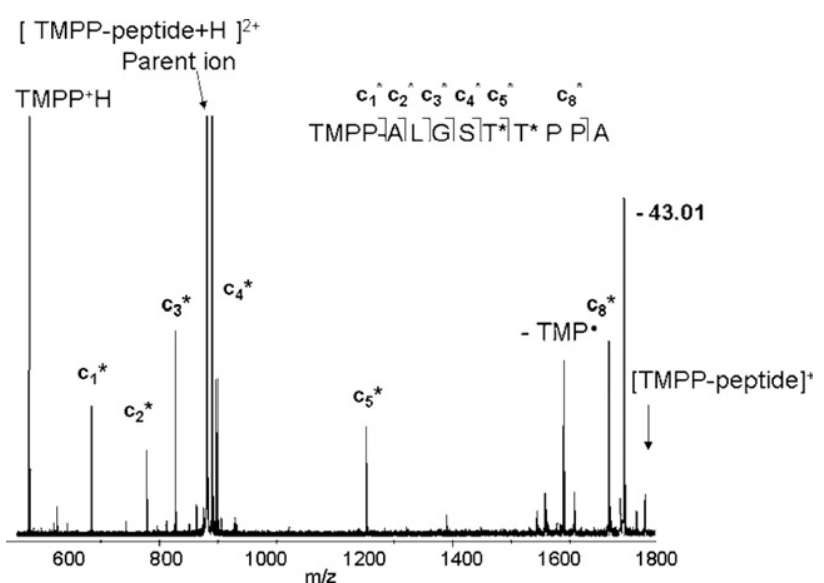

Figure 3. ECD of $2^{+}(m / z$ 896.8888) of glycosylated peptide G1 derivatized with TMPP.

group (especially when low collision energies where used), which is not the case for nonderivatized species. Similar results were obtained more recently for glycopeptides ${ }^{\circ}$ by $^{\circ} \mathrm{Czeszak}^{\circ} \mathrm{et}^{\circ}{ }^{\mathrm{al}} .^{\circ}[20]^{\circ}$ who $^{\circ}$ showed $^{\circ}$ that ${ }^{\circ}$ the MALDI-PSD spectra of $\mathrm{O}$-glycopeptides derivatized with a phosphonium allowed unambiguous assignment of the GalNAc moieties in the sequence by providing informative $a$ and $b$ ions. The main limitation of this method for sequencing glycosylated or phosphorylated peptides by SORI-CAD is that the fragments allowing the localization of the modifications in the peptides are generally of low abundance.

Since the addition of a fixed charge, such as a phosphonium group, seemed to substantially modify the behavior of peptides subjected to collision-activated dissociation, we aimed to evaluate the changes induced by such derivatization on ECD fragmentation. Ions selected in this series of experiments were mostly doubly charged, which meant that the charge carriers were a phosphonium group and a proton. For one peptide, the triply charged species (which was the most intense in the spectrum) was subjected to ECD. In that case, the charge carriers were one phosphonium and two protons.

Such ECD experiments on peptides cationized with other charge carriers than protons have previously been realized, but never with a phosphonium species. Williams et al. reported some ECD experiments on mono- or dimetalated ${ }^{\text {ppeptides }}$ 'using lithium ${ }^{\circ}{ }^{\circ}{ }^{\circ}{ }^{\circ}$ cesium [39]. They showed in their study that ECD of $[\mathrm{M}+2 \mathrm{Li}]^{2+}$ and $[\mathrm{M}+$ $2 \mathrm{Cs}]^{2+}$ ions produced mono- and dimetalated analogues of the fragment ions formed from $[\mathrm{M}+2 \mathrm{H}]^{2+}$ with the proportion of dimetalated to monometalated fragment ions increasing with fragment ion mass. For $[\mathrm{M}+\mathrm{Li}+$ $\mathrm{Cs}]^{2+}$ species, the abundance of cesiated fragment ion exceeded that of lithiated in the ECD spectra by 10:1 and all $c$ and $z$ ions formed from ECD of the $[\mathrm{M}+\mathrm{Li}+\mathrm{H}]^{2+}$ were lithiated. These results were explained by a preferred neutralization of the cation of highest recombination energy. The authors evaluated the recombination energy of protonated, lithiated, and cesiated glycine to be 116,85 , and $72 \mathrm{kcal} / \mathrm{mol}$, respectively. Zubarev et al. performed ECD on angiotensin II using $\mathrm{Zn}^{2+}$ as charge carriers and on polyglycols (PG) cationized with two sodium or two ammonium ions. For all experiments, fewer fragment ions are $^{\circ}$ observed $^{\circ}$ compared $^{\circ}$ with $^{\circ}$ protonated $^{\circ}$ species $^{\circ}[40]$. Marshall et al. showed that ECD of simultaneously protonated and cationized oligodeoxynucleotide dications yielded more product ions than that generated from the doubly ${ }^{\circ}$ protonated ${ }^{\circ}$ species $^{\circ}[41] .^{\circ}$ More $^{\circ}$ recently, ${ }^{\circ}$ divalent alkaline-earth metal ions were used as charge carriers for ECD of 'severalmodel'peptides'as'well'as'SubstancePY42]. Again, information complementary to the ECD of protonated peptides was obtained. The authors also pointed out that doubly charged molecular ions could be generated by simply doping a small amount of alkaline-earth metal salt into the analyte solution whereas doubly protonated system were sometimes difficult to attain. Similar experiments on sulfated peptides lacking basic amino acid

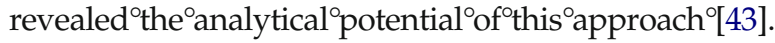

\section{TMPP-Glycopeptides}

ECD experiments on glycopeptides G1-G6 gave informative spectra containing different kind of fragment ions. The ECD spectrum obtained for the glycopeptide G1 shown ${ }^{\circ}$ in ${ }^{\circ}$ Figure $^{\circ} 3^{\circ}$ is ${ }^{\circ}$ representative ${ }^{\circ}$ of ${ }^{\circ}$ the ${ }^{\circ}$ results obtained ${ }^{\circ}$ for ${ }^{\circ}$ the ${ }^{\circ}$ glycopeptides. ${ }^{\circ}$ Note $^{\circ}$ that ${ }^{\circ}{ }^{\circ}$ Tables ${ }^{\circ} 2^{\circ}$ and 3 , ${ }^{\circ}$ respectively, ${ }^{\circ}$ gather ${ }^{\circ}$ the ${ }^{\circ}$ bond $^{\circ}{ }^{\circ}$ leavages ${ }^{\circ}$ observed ${ }^{\circ}$ for all peptides subjected to ECD and (TMPP derivatization + ECD) and the sequence coverage obtained for all these ECD experiments.

The most interesting ions observed are $c^{*}$ ions. These $c$-type ions are marked with an asterisk to indicate that the charge carrier is a phosphonium and not a proton. Their probable structure is depicted in Scheme $\mathbf{1}$. Note that no $z$ ions are observed, indicating that the charge always remains on the $\mathrm{N}$-terminus (on the phosphonium group).

These $c^{*}$ ions are of high abundance in all spectra and

Table 3. Sequence coverages for peptides G1-G6 and P1-P4 obtained for ECD experiments carried out on the native and phosphonium derivatized peptides. The sequence coverage is calculated as the ratio between the number of cleaved peptide bonds and the number of peptidic bonds in the peptide

\begin{tabular}{|c|c|c|}
\hline Peptide & $\begin{array}{c}\text { ECD sequence coverage } \\
\text { obtained on native } \\
\text { peptide }(\%)\end{array}$ & $\begin{array}{c}\text { ECD sequence coverage } \\
\text { obtained on } \\
\text { phosphonium } \\
\text { derivatized peptide (\%) }\end{array}$ \\
\hline G1 & 25 & 75 \\
\hline G2 & 37.5 & 75 \\
\hline G3 & 37.5 & 75 \\
\hline G4 & 37.5 & 75 \\
\hline G5 & 50 & 75 \\
\hline G6 & 75 & 91.5 \\
\hline P1 & 63.5 & 82 \\
\hline P2 & 72.5 & 82 \\
\hline P3 & 45.5 & 63.5 \\
\hline P4 & 50 & 87.5 \\
\hline
\end{tabular}




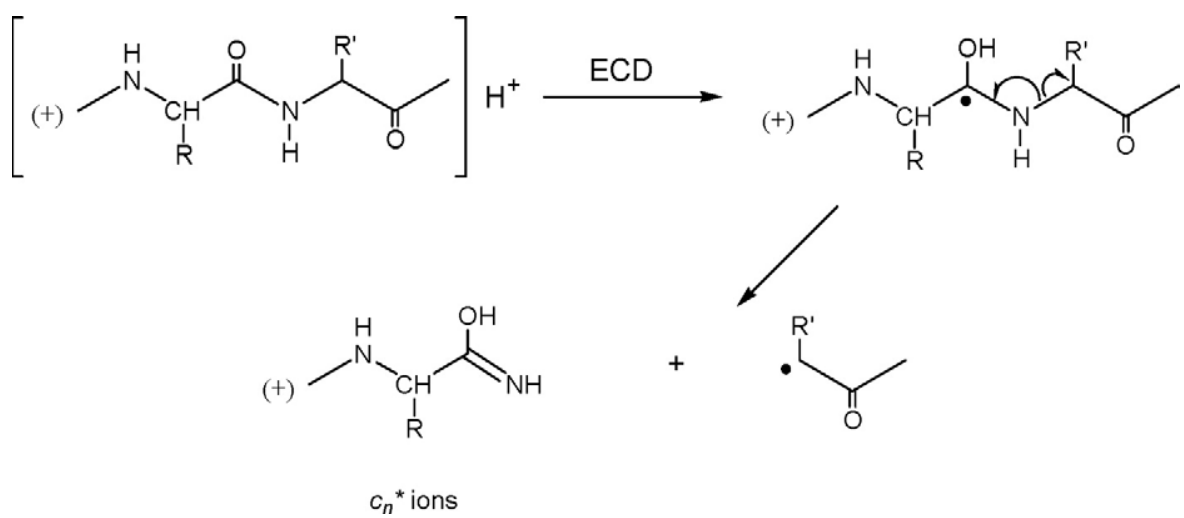

Scheme 1. Proposed structure for the $c^{*}$ ions formed from ECD of $\left(2^{+}\right)$TMPP derivatives.

may be accompanied by $\left(c^{*}-H\right)$ ions. The analysis of Table $^{\circ} 3^{\circ}$ indicates $^{\circ}$ that $^{\circ} \mathrm{TMPP}^{\circ}$ derivatization ${ }^{\circ}$ allows $^{\circ}$ a better sequence coverage in all cases. For instance, the sequence coverage of the native glycopeptide $\mathbf{G 1}$ is $25 \%$ before derivatization while of $75 \%$ after phosphonium derivatization i.e., all the peptide bonds except the Xxx-Pro linkage have been cleaved.

The exclusive presence of these $c^{*}$ ions clarifies the spectrum since it avoids the overlap of $c$ and $z$ isotopic patterns that sometimes occurs and complicates the interpretation. No loss of GalNAc was observed. For the glycopeptide G1, all $c^{*}$ ions are observed with a high abundance except $c_{6}{ }^{*}$ and $c_{7}{ }^{*}$, both corresponding to the cleavage of an Xxx-Pro bond. These results show that the presence of a fixed charge on the glycopeptides has a positive influence on the results, on sequence coverage as well as in the quality of the data obtained. Indeed, the additional mass brought by the TMPP allows the smaller $c$ ions $\left(c_{1}, c_{2}\right)$ that are rarely observed in ECD spectra to be detected easily. The presence of these ions can be of prime importance for structural purpose. In the same vein, the mass range chosen for all MS/MS spectra starts at $\mathrm{m} / \mathrm{z} 500$ instead of $\mathrm{m} / \mathrm{z} 100$, which, for a given number of data points ( $512 \mathrm{k}$ for all our experiments) increases resolution and mass accuracy.

The analysis of all ECD spectra also indicates that competitive fragmentations take place in the phosphonium moiety. These fragmentations are probably initiated by the capture of the electron by the phosphonium group (Scheme 2) to give a hypervalent phosphorus radical. The stability of hypervalent nitrogen radicals has been demonstrated some years ago by Turecek and $\operatorname{Syrstad}^{\circ}[44]^{\circ}$ and $^{\circ}$ theoretical $^{\circ}$ calculations $^{\circ}$ are $^{\circ}$ underway for phosphorus species.

This unstable species can lose a trimethoxyphenyl radical (-TMP in the spectrum) by homolytic cleavage of the $\mathrm{P}-\mathrm{C}$ bond to give a stable phosphine still bound to the protonated peptide. Another important fragmentation corresponds to the formation of the stable protonated trimethoxyphenyl phosphine at $\mathrm{m} / \mathrm{z}$ 533. This latter elimination can be rationalized by a proton transfer from the charged peptide to the phosphine moiety to give an ion-molecule complex, which easily dissociates to give the protonated phosphine. In that case, the TMPP moiety acts as a good leaving group. An attachment of a hydrogen radical to the phosphonium group followed by the cleavage of the phosphorus-methylene bond cannot be excluded.

Finally, no loss of GalNAc is observed in the spectra but the elimination of $43.01 \mathrm{Da}$ is still present in high abundance.

These results demonstrate that the addition of a phosphonium group completely modifies the reactivity

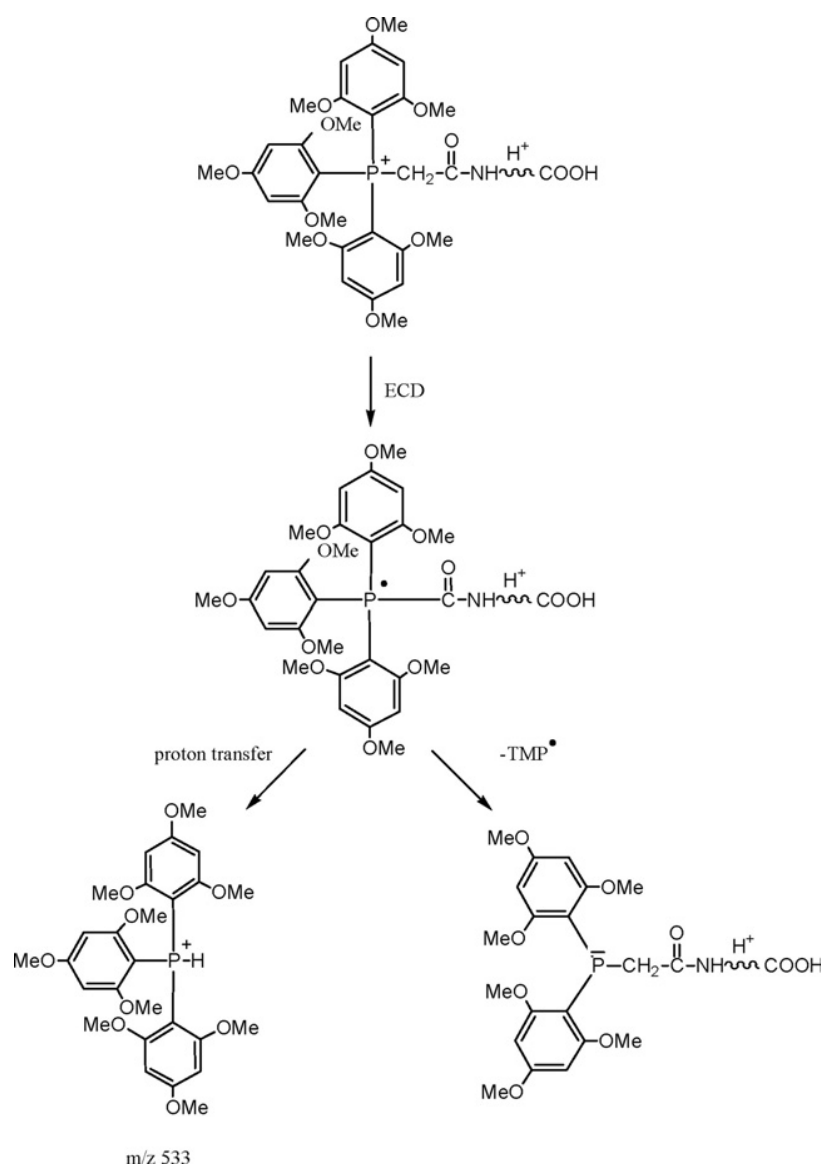

Scheme 2. Proposed structures for the fragment ions related to the TMPP moiety. 
of the glycopeptides under study. Two major factors may explain this feature. First, it has been shown that fragmentations observed in ECD of peptides strongly depend on the gas-phase structure of the precursor ions (hydrogen bonding, salt-bridge). Changing the free N-terminus for a TMPP moiety may induce an important modification of the gas-phase structure of the peptide. Second, the energetic of the electron capture is completely modified. In the native peptide, the system contains two protons, which are probably on the most basic sites of the peptide whereas the system after derivatization contains one proton and one phosphonium on the $\mathrm{N}$-terminus. The presence of regular ECD fragments ( $c^{*}$ ions) and fragment ions from the TMPP moiety clearly indicates that the recombination of the electron with the phosphonium group can compete with its recombination with the charged peptide. The improvement in sequence coverage with the $c^{*}$ ions obtained for all derivatized glycopeptides, regardless of their sequence, is probably an indication of a mechanism that does not involve directly the initial protonation site. Indeed, if the initial protonation site led to the fragmentation, the opposite trend (increased fragmentation with an increased number of protons) should be expected. Thus, these results are probably a good indication of the prevalence of a mechanism involving an electron recombination in the $\pi^{*}$ carbonyl orbitals of the polypeptide backbone rather than an alternative mechanism.

\section{TMPP-Phosphopeptides}

Similar results are obtained with phosphonium derivatized phosphopeptides since ECD spectra of all doubly charged TMPP-phosphopeptides (P1, P2, P4) contain nearly ${ }^{\circ}$ complete $^{\circ} \mathrm{C}^{*}$ ions $^{\circ}$ series $^{\circ}\left(\right.$ Table $\left.^{\circ} 2\right) .{ }^{\circ} \mathbf{P} 3,{ }^{\circ}$ for ${ }^{\circ}$ which the triply charged ion was selected for fragmentation, gives both $c^{*}$ and regular $z$ ions. These $z$ ions are due to the presence of basic residues on the C-terminus of the peptide (KKK). For example, the ECD spectrum obtained for $^{\circ}$ the ${ }^{\circ}$ phosphopeptide ${ }^{\circ} \mathbf{P} 1$ is $^{\circ}$ depicted ${ }^{\circ}{ }^{\circ}$ Figure $^{\circ} 4$.

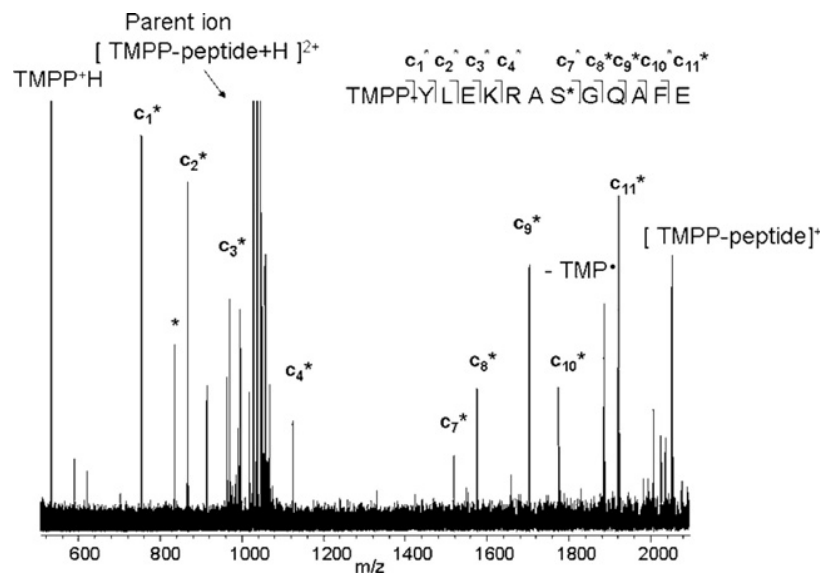

Figure 4. ECD of $2^{+}(m / z$ 1025.928) of phosphorylated peptide P1 derivatized with TMPP.
For this peptide, we observe abundant $c_{1}{ }^{*}-c_{4}{ }^{*}$ and ${c_{7}}^{*}-{c_{11}}^{*}$ series allowing an easy assignment of the phosphate in the sequence. The absence of ${c_{5}}^{*}$ and $c_{6}{ }^{* *}$ ions may be explained by the formation of a salt bridge between the negatively charged phosphate and the guanidinium moiety of the arginine. Indeed, the ECD spectrum of the doubly charged ion formed from TMPP-YLEKRASGQAFE (same sequence but no phosphate) contains $\mathcal{C}_{5}{ }^{*}$ and $c_{6}{ }^{*}$. This result indicates that the phosphate group plays an important role in the gasphase structure of the peptide and in the lack of reactivity observed in some instances. We propose that in the case of the phosphorylated peptide, the $c / z$ complex formed upon ECD does not disrupt because of a strong bonding between a positively charged arginine and a negatively charged phosphate.

The most important increase of sequence coverage can be observed for the phosphopeptide $\mathbf{P 4}$, which does not contain any basic amino acids. Moreover, the abundant losses of water observed for the native peptide are completely absent here.

As observed for TMPP-glycopeptides, ECD spectra of all TMPP-phosphopeptides exhibit a loss of a TMP radical and formation of an intense TMPP- $\mathrm{H}^{+}$ion at $m / z$ 533. No loss of phosphate is observed.

\section{Conclusions}

Our results clearly show that ECD combined with fixed charge derivatization is a powerful and new analytical tool to increase the sequence coverage of peptides bearing one or several labile post-translational modifications. A full sequence coverage is obtained for nearly all glycopeptides and phosphopeptides studied, considering that the $\mathrm{Xxx}$-Pro bond is rarely cleaved in ECD experiments. No loss of the modification is observed. In all cases, intense $c_{1}$ and $c_{2}$ ions, which very often lack in ECD spectra, can be retrieved in the spectra. The presence of these ions can be of prime importance for structural purpose. The derivatization is fast, inexpensive, and quantitative, and could thus be applied to complex mixtures such as tryptic digests. Moreover, the quality of the ECD spectra of $\left(2^{+}\right)$ions and the exclusive presence of $c^{*}$ fragments for these species can help for de novo sequencing. The difference in reactivity between protonated native peptides and their phosphonium derivatives may arise from the probable modification of the peptide structure and from the difference in energetic due to the presence of the phosphonium in the system. The presence of classical and numerous ECD fragments ( $c^{*}$ ions) and also of fragment ions originating from the TMPP moiety clearly indicate that the recombination of the electron with the phosphonium group can compete with its recombination with the $\pi^{*}$ carbonyl orbitals of the polypeptidic chain. 


\section{Acknowledgments}

The authors would like to thank C. Ganneau and V. Huteau (Institut Pasteur-Paris) for their help in the glycopeptides synthesis and P. Curmi and A. Sobel (Institut du Fer à Moulin-Paris) for the kind gift of phosphopeptides.

\section{References}

1. Huddleston, M. J.; Annan, R. S.; Bean, M. F.; Carr, S. A. Selective Detection of Phosphopeptides in Complex Mixtures by Electrospray Liquid Chromatograph/Mass Spectrometry. J. Am. Soc. Mass Spectrom. 1993, 4, 710-717.

2. Hanisch, F. G.; Green, B. N.; Bateman, R.; Peter-Katalinic, J. Localization of O-Glycosylation Sites of MUC1 Tandem Repeats by QTOF ESI Mass Spectrometry. J. Mass Spectrom. 1998, 33, 358-362.

3. Rademaker, G. J.; Pergantis, S. A.; Blok-Tip, L.; Langridge, J. I.; Kleen, A.; Thomas-Oates, J. Mass Spectrometric Determination of the Sites of O-Glycan Attachment with Low Picomolar Sensitivity. Anal. Biochem. 1998, 257, 149-160.

4. Greis, K. D.; Hayes, B. K.; Comer, F. I.; Kirk, M. K.; Barnes, S.; Lowary, T. L.; Hart, G. W. Selective Detection and Site-Analysis of O-GlcNAcModified Glycopeptides by $\beta$-Elimination and Tandem Electrospray Mass Spectrometry. Anal. Biochem. 1996, 234, 38-49.

5. Mirgorodskaya, E.; Hassan, H.; Clausen, H.; Roepstorff, P. Mass Spectrometric Determination of O-Glycosylation Sites Using $\beta$-Elimination and Partial Acid Hydrolysis. Anal. Chem. 2001, 73, 1263-1269.

6. Mirgorodskaya, E.; Roepstorff, P.; Zubarev, R. A. Localization of O-Glycosylation Sites in Peptides by Electron Capture Dissociation in a Fourier Transform Mass Spectrometer. Anal. Chem. 1999, 71, 4431-4436.

7. Cooper, H.; Håkansson, K.; Marshall, A. G. The Role of Electron Capture Dissociation in Biomolecular Analysis. Mass Spectrometry. Reviews 2005, 24, 201-222.

8. Syka, J. E. P.; Coon, J. J.; Schroeder, M. J.; Shabanowitz, J.; Hunt, D. Peptide and Protein Sequence Analysis by Electron Transfer Dissociation Mass Spectrometry. PNAS 2004, 26, 9528-9533.

9. Shi, S. D.-H.; Hemling, M. E.; Carr, S. A.; Horn, D. M.; Lindgh, I.; McLafferty, F. W. Phosphopeptide/Phosphoprotein Mapping by Electron Capture Dissociation Mass Spectrometry. Anal. Chem. 2001, 73, $19-22$

10. Kelleher, N. L.; Zubarev, R. A.; Bush, K.; Furie, B.; Furie, B. C.; McLafferty, F. W.; Walsh, C. T. Localization of Labile Posttranslational Modifications by Electron Capture Dissociation: The Case of $\gamma$-Carboxyglutamic Acid. Anal. Chem. 1999, 71, 4250-4253.

11. Ge, Y.; Lawhorn, B. G.; El Naggar, M.; Strauss, E.; Park, J.-H.; Begley, T. P.; McLafferty, F. W. Top Down Characterization of Larger Proteins (45 kDa) by Electron Capture Dissociation Pass Spectrometry. J. Am. Chem. Soc. 2002, 124, 672-678.

12. Bogdanov, B.; Smith, R. D. Proteomics by FTICR Mass Spectrometry: Top Down and Bottom Up. Mass Spectrom. Rev. 2005, 24, 168-200.

13. O'Connor, P. B.; Lin, C.; Cournoyer, J. J.; Pittman, J. L.; Belyayev, M.; Budnik, B. A. Long-Lived Electron Capture Dissociation Product Ions Experience Radical Migration via Hydrogen Abstraction. J. Am. Soc. Mass Spectrom. 2006, 17, 576-585.

14. Savitski, M. M.; Kjeldsen, F.; Nielsen, M. L.; Zubarev, R. A. Hydrogen Rearrangement to and from Radical z-Fragments in Electron Capture Dissociation of Peptides. J. Am. Soc. Mass Spectrom. 2007, 18, 113-120.

15. Horn, D. M.; Ge, Y.; McLafferty, F. W. Activated Ion Electron Capture Dissociation for Mass Spectral Sequencing of Larger (42 kDa) Proteins. Anal. Chem. 2000, 72, 4778-4784.

16. Han, X.; Jin, M.; Breuker, K.; McLafferty, F. W. Extending Top-Down Mass Spectrometry to Proteins with Masses Greater than $200 \mathrm{kDa}$. Science 2006, 314, 109-112.

17. Chalmers, M. J.; Håkansson, K.; Johnson, R.; Smith, R.; She, J.; Emmett, M. R.; Marshall, A. G. Protein Kinase A Phosphorylation Characterized by Tandem Fourier Transform Ion Cyclotron Resonance Mass Spectrometry. Proteomics 2004, 4, 970-981.

18. Håkansson, K.; Chalmers, M. J.; Quinn, J. P.; McFarland, M. A; Hendrickson, C. L.; Marshall, A. G. Combined Electron Capture and Infrared Multiphoton Dissociation for Multistage MS/MS in a Fourier Transform Ion Cyclotron Resonance Mass Spectrometer. Anal. Chem. 2003, 75, 3256-3262.

19. Sadagopan, N.; Malone, M.; Watson, J. T. Effect of Charge Derivatization in the Determination of Phosphorylation Sites in Peptides by Electrospray Ionization Collision-activated Dissociation Tandem Mass Spectrometry. J. Mass Spectrom. 1999, 34, 1279-1282.
20. Czeszak, X.; Morelle, W.; Ricart, G.; Tetaert, D.; Lemoine, J. Localization of the O-Glycosylated Sites in Peptides by Fixed-Charge Derivatization with a Phosphonium Group. Anal. Chem. 2004, 76, 4320-4324.

21. Caravatti, P.; Allemann, M. The Infinity Cell-A New Trapped-Ion Cell with Radiofrequency Covered Trapping Electrodes for FourierTransform Ion-Cyclotron Resonance Mass-Spectrometry. Org. Mass Spectrom. 1991, 26, 514-518.

22. Gauthier, J. W.; Trautman, T. R.; Jacobson, D. B. Sustained OffResonance Irradiation for Collision-Activated Dissociation Involving Fourier Transform Mass Spectrometry. Collision-Activated Dissociation Technique that Emulates Infrared Multiphoton Dissociation. Anal. Chim. Acta 1991, 246, 211-225.

23. Tsybin, Y. O.; Ramström, M.; Witt, M.; Baykut, G.; Håkansson, P. Peptide and Protein Characterization by High-Rate Electron Capture Dissociation Fourier Transform Ion Cyclotron Resonance Mass Spectrometry. J. Mass Spectrom. 2004, 39, 719-729.

24. Zubarev, R. A.; Kelleher, N. L.; McLafferty, F. W. Electron Capture Dissociation of Multiply Charged Protein Cations. A Nonergodic Process. J. Am. Chem. Soc. 1998, 120, 3265-3266.

25. Cooper, H. J. Investigation of the Presence of $b$ Ions in Electron Capture Dissociation Mass Spectra. J. Am. Soc. Mass Spectrom. 2005, 16, 19321940.

26. Haselmann, K. F.; Schmidt, M. Do b-Ions Occur from Vibrational Excitation Upon H-Desorption in Electron Capture Dissociation? Rapid Commun. Mass Spectrom. 2007, 21, 1003-1008.

27. Mormann, M.; Paulsen, H.; Peter-Katalinic, J. Electron Capture Dissociation of O-Glycosylated Peptides: Radical Site-Induced Fragmentation of Glycosidic Bond. Eur. J. Mass Spectrom. $112005497-511$.

28. Syrstad, E. A.; Turecek, F. Toward a General Mechanism of Electron Capture Dissociation. J. Am. Soc. Mass Spectrom. 2005, 16, 208-224.

29. Anusiewicz, I.; Berdys-Kochanska, J.; Skurski, P.; Simons J. Simulating Electron Transfer Attachment to a Positively Charged Model Peptide. J. Phys. Chem. A 2006, 110, 1261-1266.

30. Anusiewicz, I.; Berdys-Kochanska, J.; Simons, J. The Electron Attachment Step in Electron Capture (ECD) and Electron Transfer Dissociation (ETD). J. Phys. Chem. A 2005, 109, 5801-5813.

31. Sobczyk, M.; Simons, J. Distance Dependence of Through-Bond Electron Transfer Rates in Electron-Capture and Electron-transfer Dissociation. Int. J. Mass Spectrom. 2006, 253, 274-280.

32. Sobczyk, M.; Simons, J. The Role of Excited Rydberg States in Electron Transfer Dissociation. J. Phys. Chem. B 2006, 110, 7519-7527.

33. Huang, Z. H; Wu, J.; Gage, D. A.; Watson, J. T. A Picomole-Scale Method for Charge Derivatization of Peptides for Sequence Analysis by Mass Spectrometry. Anal. Chem. 1997, 69, 137-144.

34. Sadagopan, N.; Watson, J. T. Investigation of the Tris(Trimethoxyphenyl)Phosphonium Acetyl Charged Derivatives of Peptides by Electrospray Ionization Mass Spectrometry and Tandem Mass Spectrometry. J. Am. Soc. Mass Spectrom. 2000, 11, 107-119.

35. Huang, Z. H; Shen, T.; Wu, J.; Gage, D. A.; Watson, J. T. Protein Sequencing by Matrix-Assisted Laser Desorption Ionization-Postsource Decay-Mass Spectrometry Analysis of the N-Tris(2,4,6-Trimethoxyphenyl)PhosphineAcetylated Tryptic Digests. Anal. Biochem. 1999, 268, 305-317.

36. Roth, K. D. W.; Huang, Z. H.; Sadagopan, N.; Watson, J. T. Charge Derivatization of Peptides for Analysis by Mass Spectrometry. Mass Spectrom. Rev. 1998, 17, 255-274.

37. Sadagopan, N.; Watson, J. T. Mass Spectrometric Evidence for Mechanisms of Fragmentation of Charge-Derivatized Peptides. J. Am. Soc. Mass Spectrom. 2001, 12, 399-409.

38. Chen, W.; Lee, P. J.; Shion, H.; Ellor, N.; Gebler, J. C. Improving de Novo Sequencing of Peptides Using a Charged Tag and C-Terminal Digestion. Anal. Chem. 2007, 79, 1583-1590.

39. Iavarone, A. T.; Paech, K.; Williams, E. R. Effects of Charge and Cationizing Agent on the Electron Capture Dissociation of a Peptide. Anal. Chem. 2004, 76, 2231-2238.

40. Zubarev, R. A.; Haselmann, K. F.; Budnik, B.; Kjeldsen, F.; Jensen, F.Towards an Understanding of the Mechanism of Electron-Capture Dissociation: A Historical Perspective and modern ideas. Eur. J. Mass Spectrom. 2002, 8, 337-349.

41. Håkansson, K.; Hudgins, R. R.; Marshall, A. G. Electron Capture Dissociation and Infrared Multiphoton Dissociation of Oligodeoxynucleotide Dications. J. Am. Soc. Mass Spectrom. 2003, 14, 23-41.

42. Fung, Y. M. E.; Liu, H.; Chan, T.-W. D. Electron Capture Dissociation of Peptides Metalated with Alkaline-Earth Metal Ions. J. Am. Soc. Mass Spectrom. 2006, 17, 757-771.

43. Liu, H.; Håkansson, K. Electron Capture Dissociation of Tyrosine O-Sulfated Peptides Complexed with Divalent Metal Cations. Anal. Chem. 2006, 78, 7570-7576.

44. Shaffer, S. A.; Turecek, F. Hydrogentrimethylammonium: A marginally stable hypervalent radical. J. Am. Chem. Soc. 1994, 116, 8647-8653. 